\title{
Caracterización clínica, serológica y patrón radiológico de una cohorte unicéntrica de pacientes con enfermedad pulmonar difusa
}

\author{
JOSÉ MIGUEL CHAHUÁN S.*, MARÍA JESÚS FUENZALIDA L.**, PABLA CATALDO V.*, \\ MACARENA LAGOS C.**, ISABEL DE LA FUENTE M.***, GONZALO PEREIRA R.****, \\ JOSÉ PIZARRO P.**** y MATÍAS FLORENZANO V.*****
}

Clinical, serological and radiological pattern characterization of a monocentric cohort of patients with diffuse lung disease

Diffuse Lung Disease (DLD) is an important cause of morbidity and mortality, however in Chile we are lacking of epidemiological data on this condition. Our aim is communicate the first report of a prospective registry of DLD patients treated at bronchopulmonary unit of DIPRECA hospital. Methods: Cross-sectional study in patients referred to our bronchopulmonary unit under suspicion of DLD. Diagnosis was confirmed by chest computed tomography and informed consent was approved by patients. Data regarding clinical, serological, pulmonary function tests and echocardiography were collected from 2014 up to date. Results: 30 patients were analysed, their median of age was 76.5 yearsold (Interquartile Range 68-80), 56.7\% were women, median duration of disease: 4 years (IQR 1-10.6) and $43 \%$ has smoking history. Most frequent signs and symptoms were crackles (97\%), dyspnoea (90\%) and cough (57\%). Comorbidities: $3 \%$ had asthma, 3\% chronic obstructive pulmonary disease and $6.7 \%$ connective tissue diseases. Radiological findings: $20 \%$ had DLD with usual interstitial pneumonia pattern (UIP), 23\% DLD possible UIP, 30\% DLD inconsistent with UIP, 14\% chronic hypersensitivity pneumonitis and 13\% nonspecific interstitial pneumonia. Serology: 18\% had positive rheumatoid factor of which only one case had rheumatoid arthritis, 67\% had positive antinuclear antibodies (ANA), 17\% ANCA positive of which only one case of clinical vasculitis. Spirometry was mainly normal (52\%) or restrictive (45\%). Echocardiography showed pulmonary hypertension mainly mild in $52 \%$ of patients. No significant association was found between titles of ANA $\geq 1 / 320$ and gender, smoking or radiological pattern. Conclusions: Our demographic and radiological findings are similar to those reported in literature; however, the highlights in our cohort are the increased frequency of female gender and positive ANA without history or clinical manifestation of connective tissue diseases.

Key words: Idiopathic pulmonary fibrosis, tomography X-Ray computed, female, antibodies, antinuclear, cross-sectional studies.

\section{Resumen}

La enfermedad pulmonar difusa (EPD) es causa importante de morbimortalidad; a pesar de esto no tenemos datos epidemiológicos en Chile. Nuestro objetivo es comunicar el primer reporte del registro prospectivo de pacientes con EPD atendidos en la unidad de broncopulmonar del hospital DIPRECA. Métodos: Estudio de corte transversal en pacientes derivados al policlínico broncopulmonar del hospital DIPRECA por sospecha de EPD. En caso de confirmación diagnóstica por tomografía computada de tórax y consentimiento informado aprobado por los pacientes, se compilaron datos clí-

\footnotetext{
* Residente Medicina Interna, Universidad de Valparaíso.

** Residente Medicina Interna, Universidad Diego Portales.

*** Unidad de Broncopulmonar Hospital DIPRECA.

**** Unidad de Radiología Hospital DIPRECA (Dirección de Previsión de Carabineros de Chile). Las Condes, RM.

*****Instituto Nacional del Tórax, Providencia, Santiago, Chile.
} 
nicos, serológicos, pruebas de función pulmonar y ecocardiografía, desde 2014 hasta la fecha. Resultados: Se analizaron 30 pacientes la mediana de su edad fue 76,5 años (rango intercuartílico 68-80), $56,7 \%$ eran mujeres, duración mediana de la enfermedad: 4 años (RIC 1-10,6) y 43\% con antecedentes de tabaquismo. Los síntomas y signos más frecuente fueron crujidos (97\%), disnea (90\%) y tos (57\%). Comorbilidades: $3 \%$ tenía asma, 3\% enfermedad pulmonar obstructiva crónica y 6,7\% enfermedades del tejido conectivo. Hallazgos radiológicos: $20 \%$ tenía EPD con patrón de neumonía intersticial usual (UIP), 23\% EPD posible UIP, 30\% EPD inconsistente con UIP, 14\% neumonitis por hipersensibilidad crónica y 13\% neumonía intersticial no especifica. Serología: 18\% tenía factor reumatoide positivo de ellos sólo uno de los casos tenía artritis reumatoide, el 67\% tenía anticuerpos antinucleares (ANA) positivos, $17 \%$ ANCA positivo de ellos sólo un caso tenía historia de vasculitis clínica. La espirometría fue mayoritariamente normal (52\%) o restrictiva (45\%). Ecocardiografía detectó hipertensión pulmonar mayoritariamente leve en $52 \%$ de los pacientes. No se encontró asociación significativa entre los títulos de ANA $\geq 1 / 320$ en relación a género, tabaquismo o patrón radiológico. Conclusiones: Nuestros hallazgos demográficos y radiológicos son similares a los de la literatura; sin embargo, destaca en nuestra cohorte la mayor frecuencia de género femenino y ANA positivos sin historia o manifestación clínica de enfermedades del tejido conectivo.

Palabras clave: Fibrosis pulmonar idiopática, tomografía computarizada, género femenino, anticuerpos antinucleares, estudios de corte transversal.

\section{Introducción}

La enfermedad pulmonar difusa (EPD) es un término general que comprende más de 200 patologías pulmonares y sistémicas que se caracterizan ya sea por inflamación y/o fibrosis del parénquima alveolar, siendo la fibrosis pulmonar idiopática (FPI) la más común de ellas ${ }^{1}$ con una incidencia estimada en 0,22 a 8,8 por 100.000 habitantes $^{2}$. Si bien en forma individual la mayoría de las EPD son infrecuentes, como grupo colectivo son causa importante de morbilidad y mortalidad estimándose más de 50 millones de afectados en el mundo y hasta el 15\% de los motivos de consulta a neumólogos. En Chile existe un registro nacional en curso, sin embargo, hasta el momento no contamos con una descripción epidemiológica.

Desde el año 2014 a la fecha, en el hospital DIPRECA hemos llevado a cabo un registro de los pacientes con EPD atendidos en la unidad de broncopulmonar. El propósito de este documento es presentar el primer reporte de este registro con el fin de contribuir a la caracterización epidemiológica de este grupo de enfermedades en la realidad chilena.

\section{Método}

En el año 2014 diseñamos un flujograma de derivación al policlínico de broncopulmonar de nuestro hospital de pacientes con sospecha de EPD expuesto en la Figura 1. Aquellos pacientes con criterios de sospecha de EPD se les solicita estudio inicial con tomografía computada de tó-
$\operatorname{rax}(\mathrm{TC}-\mathrm{Tx})$, que son analizados por radiólogos expertos en TC-Tx, y luego son derivados a dicho policlínico donde son evaluados por neumólogos. En caso de evaluación TC-Tx compatible con EPD previo consentimiento informado aprobado por el paciente, se registraron datos demográficos y clínicos en una cartola diseñada para este fin continuando con el estudio inicial consistente en estudio de función pulmonar con espirometría y test de difusión de monóxido de carbono $\left(\mathrm{DL}_{\mathrm{CO}}\right)$, ecocardiograma y estudio inmunológico con factor reumatoide (FR), anticuerpos antinucleares (ANA), antígenos extraíbles del núcleo (ENA), anti-DNA, y anticuerpos anticitoplasma de neutrófilos (ANCA). Respecto a los parámetros de medición de las variables evaluadas, la disnea fue objetivada mediante escala modificada del Medical Research Council (mMRC). La presencia de hipertensión pulmonar se evaluó según estimación de la presión sistólica de arteria pulmonar (PSAP) considerándose leve: PSAP 35-50 $\mathrm{mmHg}$; moderada $51-70 \mathrm{mmHg}$ y severa $\geq 71 \mathrm{mmHg}$. Finalmente todos estos datos fueron tabulados en tabla Excel.

A partir de esta base de datos y previa aprobación por el comité de ética de nuestro hospital, se realizó un estudio de corte transversal descriptivo y analítico. El análisis estadístico descriptivo se realizó con frecuencias (porcentajes) y medianas (rango intercuartílico). Se evaluó si existía asociación en pacientes con títulos de ANA $\geq 1 / 320$ respecto a género, antecedente de tabaquismo y patrón radiológico mediante test de Fisher considerando un $\mathrm{p}<0,05$ para indicar significancia estadística. 


\section{FLUJOGRAMA}

\section{POLLCLINICO/SERVICIO MEDICINA}

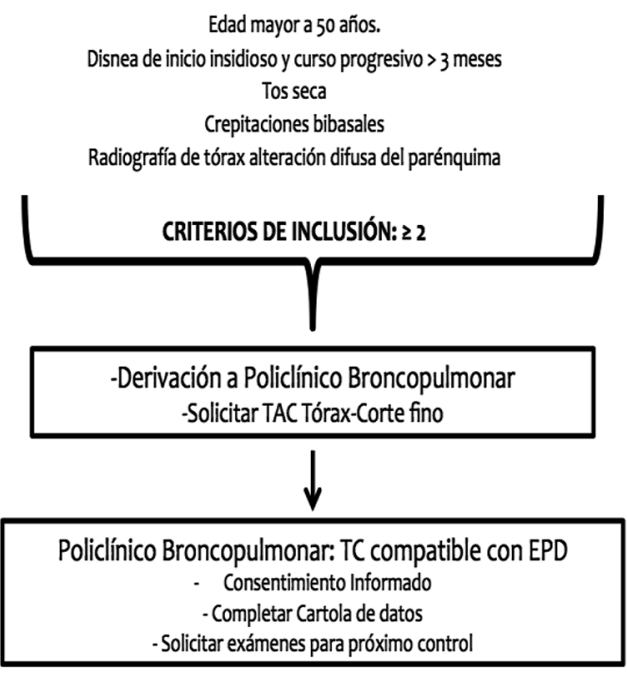

Figura 1. Flujograma de derivación de pacientes con sospecha de enfermedad pulmonar difusa.

\section{Resultados}

Se analizaron 30 pacientes del registro. De estos pacientes, la mediana de edad fue de 76,5 años (rango intercuartílico 68-80), el 56,7\% mujeres y la duración mediana de la enfermedad fue de 4 años (RIC 1-10,6). El 43\% tenía antecedentes de tabaquismo y $20 \%$ antecedente familiar de primer grado de EPD. Respecto a síntomas y signos un $57 \%$ refirió tos crónica, un $90 \%$ disnea siendo $m M R C \geq 2$ en el $73 \%$ de los casos, $43 \%$ pirosis, $97 \%$ crujidos y $13 \%$ acropaquia. En cuanto a comorbilidades 3\% tenía asma, 3\% enfermedad pulmonar obstructiva crónica y $6,7 \%$ enfermedades del tejido conectivo consistentes en un caso de artritis reumatoide (AR) y un caso de vasculitis ANCA-MPO. Al estudio radiológico se encontró $20 \%$ con patrón de neumonía intersticial usual (UIP), $23 \%$ posible UIP, 30\% inconsistente con UIP, $14 \%$ neumonitis por hipersensibilidad crónica $(\mathrm{NH})$ y $13 \%$ neumonía intersticial no específica (NSIP). En cuanto a las pruebas de función pulmonar, la espirometría fue normal $(52 \%)$ o restrictiva $(45 \%)$ en la mayoría de los casos, sólo un caso presentó un patrón obstructivo correspondiente a un paciente con antecedentes de EPOC y patrón radiológico UIP; en cuanto al $\mathrm{DL}_{\mathrm{CO}}$, ésta se logró medir en 25 pacientes de los cuales la mayoría presentó disminución de la difusión (88\%) principalmente en rango leve (44\%); $16 \%$ tenían disminución severa de $\mathrm{DL}_{\mathrm{CO}}$, en este subgrupo todos presentaron patrón radiológico de tipo UIP o posible UIP, sin embargo, sin correlación con el grado de severidad de la alteración espirométrica. Al estudio con ecocardiograma el $52 \%$ de los pacientes cumplieron criterios de hipertensión pulmonar siendo leve en la mayoría de los casos (Tabla 1).

Sólo en dos casos se hizo estudio histológico: un paciente con patrón radiológico de $\mathrm{NH}$ en que la histología reveló pérdida de la arquitectura pulmonar por un proceso fibroso intersticial con infiltrado inflamatorio mixto y focos de proliferación fibroblástica asociado a bronquiectasias sugerente de UIP; y un paciente con patrón radiológico UIP en estudio pre trasplante cuya histología confirmo dicho patrón.

Al estudio de autoinmunidad se obtuvo FR en 28 pacientes con un $18 \%$ con FR positivo de los cuales sólo uno de ellos tenía antecedente de AR presentando al TC-Tx patrón de UIP, los otros pacientes con FR $(+)$ correspondieron a un caso con UIP, un caso posible UIP y dos casos con patrón de NH. Se obtuvo medición de ANA en 27 pacientes con $67 \%$ de casos positivos siendo el patrón homogéneo el más frecuente $(72 \%)$ y observándose títulos $\geq 1 / 320$ en el $56 \%$; todos los ENA fueron negativos. El estudio de los ANCA se realizó en 19 pacientes siendo positivo en 17\% de ellos correspondiente a un caso de vasculitis ANCA-MPO y patrón de UIP, los dos casos restantes correspondieron a un caso de paciente con AR con MPO $(+)$ y patrón UIP y un caso PR3 $(+)$ con patrón NSIP, ambos sin historia de vasculitis (Tabla 2).

No se encontró asociación significativa entre los títulos de ANA $\geq 1 / 320$ con respecto al género $(\mathrm{p}=0,63)$, tabaquismo $(\mathrm{p}=0,13)$ o patrón radiológico $(\mathrm{p}=1,0)$.

\section{Comentarios}

Este trabajo fue realizado con el fin de identificar las principales características de la EPD en pacientes chilenos, siendo uno de los primeros estudios descriptivos a nivel nacional en reportar sus registros. Se realizó una evaluación de las principales características clínicas, serológicas, radiológicas y de función pulmonar implicadas tanto en el estudio etiológico como en la evaluación de factores pronósticos relacionados a este grupo de enfermedades.

Encontramos como patrón radiológico más frecuente a la EPD con patrón consistente/po- 
Tabla 1. Características de los 30 pacientes con enfermedad pulmonar difusa ingresados al estudio

\begin{tabular}{|c|c|c|c|c|c|c|}
\hline \multirow[t]{4}{*}{ Demográficas } & Mujeres & $\mathrm{n}(\%)$ & 17 & $(56,7)$ & & \\
\hline & Edad mediana en años & (RIC) & 76,5 & $(68-80$ & & \\
\hline & Tabaquismo & $\mathrm{n}(\%)$ & 13 & $(43,3)$ & & \\
\hline & Antecedente familiar $1^{\text {er }}$ grado EPD* & $\mathrm{n}(\%)$ & 6 & $(20 \%)$ & & \\
\hline \multirow[t]{9}{*}{ Clínicas } & Duración mediana de la enfermedad en años & (RIC) & 4 & $(1-10,6$ & & \\
\hline & Tos crónica & $\mathrm{n}(\%)$ & 17 & $(56,6)$ & & \\
\hline & Disnea $^{\dagger}$ & $\mathrm{n}(\%)$ & 27 & $(90)$ & \multicolumn{2}{|c|}{$\begin{array}{l}\operatorname{mMRC} 0=3(10) \\
\operatorname{mMRC} 1=5(16,6) \\
\operatorname{mMRC} 2=5(16,6) \\
\text { mMRC } 3=12(40) \\
\text { mMRC } 4=3(16,6)\end{array}$} \\
\hline & Pirosis & $\mathrm{n}(\%)$ & 13 & \multicolumn{3}{|c|}{$(43,3 \%)$} \\
\hline & Crujidos & $\mathrm{n}(\%)$ & 29 & \multicolumn{3}{|c|}{$(96,6 \%)$} \\
\hline & Acropaquia & $\mathrm{n}(\%)$ & 4 & \multicolumn{3}{|l|}{$(13,3)$} \\
\hline & Antecedente de asma & $\mathrm{n}(\%)$ & 1 & \multicolumn{3}{|c|}{$(3,3 \%)$} \\
\hline & Antecedente de EPOC & $\mathrm{n}(\%)$ & 1 & \multicolumn{3}{|c|}{$(3,3 \%)$} \\
\hline & Antecedente de enfermedad del tejido conectivo & $\mathrm{n}(\%)$ & 2 & \multicolumn{3}{|c|}{$\begin{array}{l}(6,7) 1 \text { caso } \mathrm{AR}^{\S} \\
1 \text { caso vasculitis ANCA }\end{array}$} \\
\hline \multirow[t]{5}{*}{ Radiológicas } & UIP & $\mathrm{n}(\%)$ & 6 & \multicolumn{3}{|l|}{$(20)$} \\
\hline & Posible UIP & $\mathrm{n}(\%)$ & 7 & \multicolumn{3}{|l|}{$(23,3)$} \\
\hline & Inconsistente con UIP & $\mathrm{n}(\%)$ & 9 & \multicolumn{3}{|l|}{$(30)$} \\
\hline & $\mathrm{NH}$ & $\mathrm{n}(\%)$ & 4 & \multicolumn{3}{|l|}{$(13,3)$} \\
\hline & NSIP & $\mathrm{n}(\%)$ & 4 & \multicolumn{3}{|l|}{$(13,3)$} \\
\hline \multirow[t]{2}{*}{ Función pulmonar } & Espirometría & $\mathrm{n}(\%)$ & \multicolumn{3}{|c|}{$\begin{array}{l}\text { Normal } \\
\text { Restrictiva } \\
\text { Obstructiva }\end{array}$} & $\begin{array}{l}15(50) \\
14(46,7) \\
1(3,3)\end{array}$ \\
\hline & $\mathrm{DL}_{\mathrm{CO}}$ & $\mathrm{n}(\%)$ & \multicolumn{3}{|c|}{$\begin{array}{l}\text { Normal } \\
\text { Disminución Leve } \\
\text { Disminución Moderada } \\
\text { Disminución Severa }\end{array}$} & $\begin{array}{r}3(12) \\
11(44) \\
7(28) \\
4(16)\end{array}$ \\
\hline Ecocardiograma & Hipertensión Pulmonar** & $\mathrm{n}(\%)$ & \multicolumn{3}{|c|}{$\begin{array}{l}\text { Ausente } \\
\text { Leve } \\
\text { Moderada } \\
\text { Severa }\end{array}$} & $\begin{array}{r}14(48,3) \\
11(37,9) \\
3(10,3) \\
1(3,4)\end{array}$ \\
\hline
\end{tabular}

*EPD: Enfermedad Pulmonar Difusa; †Disnea según escala modificada del Medical Research Council (mMRC); "EPOC: Enfermedad pulmonar obstructiva crónica; ${ }^{\S} \mathrm{AR}$ : Artritis reumatoide; ${ }^{\text {DL }}$ Co según \% del valor teórico: leve ( $>60 \%$ y $<$ límite inferior normalidad); moderada $(>40 \%-60 \%)$ y severa $(<40 \%)$. $* *$ Hipertensión pulmonar estimada por presión sistólica de arteria pulmonar (PSAP): leve (35-50 mmHg); moderada (51-70 mmHg) y severa $(\geq 71 \mathrm{mmHg})$. RIC: rango intercuartil. ANCA: Anticuerpos anticitoplasma de neutrófilos.

sible con UIP en un $43,3 \%$, lo cual es similar a lo reportado en series clínicas internacionales ${ }^{3}$. Respecto a las características demográficas, datos similares se describen en cuanto a la edad de presentación y el antecedente de EPD en familiares de primer grado ${ }^{4}$. Sin embargo, a diferencia de lo clásicamente descrito, en nuestro estudio se observó un mayor porcentaje de casos en el género femenino alcanzando el $56,7 \%$ del total de la muestra, lo cual coincide con el pre-informe de la serie nacional entregada en el congreso ALAT 2016.

De igual forma el hábito tabáquico fue menos frecuente que lo reportado en otros estudios ${ }^{5}$. Con respecto a los aspectos clínicos son similares a los descritos, siendo la presencia de crujidos, tos y disnea los hallazgos más frecuentes.

En el estudio de función pulmonar el hallazgo más común fue una $\mathrm{DL}_{\mathrm{CO}}$ disminuida, en contraste con la espirometría que fue normal en la 
Tabla 2. Relación entre serología autoinmune y patrón radiológico en pacientes con enfermedad pulmonar difusa

\begin{tabular}{|ccccl|}
\hline Paciente & FR & ANA $\geq \mathbf{3 2 0}$ & ANCA & Patrón radiológico \\
\hline 1 & $(+)$ & $(-)$ & $(-)$ & UIP \\
\hline $2^{*}$ & $(+)$ & $(-)$ & MPO & UIP \\
\hline 3 & $(+)$ & $1 / 640$ moteado & $(-)$ & NH \\
\hline 4 & $(+)$ & $(-)$ & $(-)$ & Posible UIP \\
\hline 5 & $(+)$ & $(-)$ & $(-)$ & NH \\
\hline 6 & $(-)$ & $1 / 320$ moteado & $(-)$ & Posible UIP \\
\hline 7 & $(-)$ & $1 / 320$ homogéneo & $(-)$ & Inconsistente UIP \\
\hline 8 & $(-)$ & $1 / 640$ moteado & $(-)$ & NSIP \\
\hline 9 & $(-)$ & $1 / 1.280$ moteado & $(-)$ & NSIP \\
10 & $(-)$ & $1 / 320$ homogéneo & $(-)$ & Inconsistente UIP \\
\hline 11 & $(-)$ & $1 / 640$ moteado & $(-)$ & Posible UIP \\
12 & $(-)$ & $1 / 320$ nucleolar & $(-)$ & Inconsistente UIP \\
\hline 13 & $(-)$ & $1 / 320$ moteado & $(-)$ & NH \\
14 & $(-)$ & $1 / 320$ moteado & $(-)$ & Inconsistente UIP \\
\hline 15 & $(-)$ & $(-)$ & PR3 & NSIP \\
\hline 16 & $(-)$ & $(-)$ & MPO & Posible UIP \\
\hline
\end{tabular}

*Paciente con antecedente de artritis reumatoide; ${ }^{\dagger}$ Paciente con antecedentes de vasculitis ANCA-MPO; FR: Factor reumatoideo; ANA: anticuerpos antinucleares; ANCA: Anticuerpos anticitoplasma de neutrófilos; MPO: Mieloperoxidasa; PR3: proteinasa 3. La abreviaturas de los patrones radiológicos están especificadas en el texto.

mitad de los casos. Respecto a los pacientes con alteración espirométrica el patrón restrictivo fue lo más observado como era de esperarse, presentando patrón obstructivo sólo un paciente que tenía antecedente previo de EPOC. En cuanto a la presencia de hipertensión pulmonar, si bien es una complicación frecuente reportándose en el $30-40 \%$ de los pacientes con $\mathrm{EPD}^{6}$, destaca la mayor frecuencia de este hallazgo en nuestra muestra correspondiente al $52 \%$ de los casos. Sin embargo, debe tenerse en cuenta que su estimación sólo se realizó mediante ecocardiograma y no consideramos en el registro comorbilidades frecuentes en adultos mayores como es la presencia de insuficiencia cardiaca que pudiese haber contribuido en la mayor frecuencia de este hallazgo.

En el estudio de autoinmunidad dos pacientes tenían el antecedente previo de enfermedades autoinmunes. Un paciente con AR con FR (+) y MPO (+) presentando EPD con patrón UIP lo cual corresponde al compromiso pulmonar de tipo intersticial más frecuentemente descrito en esta enfermedad ${ }^{7}$. El otro caso corresponde a un paciente con vasculitis ANCA MPO con patrón de EPD posible UIP; respecto a este hecho existen estudios que han reportado asociación entre EPD particularmente con patrón UIP y la presencia de MPO $(+)$, incluso en pacientes sin antecedente de poliangeítis microscópica ${ }^{8}$. Sin embargo, se debe considerar que esta asociación aún no está del todo elucidada y no forma parte de los exámenes iniciales descritos en cuanto al estudio de autoinmunidad en pacientes EPD.

Los hallazgos más relevantes de nuestro registro corresponden a la alta frecuencia de pacientes con ANA $(+)$ incluso con títulos $\geq 1 / 320$, destacando que la mayoría de los pacientes no tenían antecedentes previos de enfermedades del tejido conectivo. A pesar de la alta frecuencia no se encontró asociación significativa entre los títulos de ANA $\geq 1 / 320$ con respecto al género, tabaquismo ni patrón radiológico. Estos hallazgos cobran mayor relevancia teniendo en cuenta la reciente publicación en el año 2015 realizada en conjunto por la ERS (European Respiratory Society) y ATS (American Thoracic Society) ${ }^{9}$ respecto a la introducción del concepto de "neumonía intersticial con características autoinmunes" con criterios definidos según los cuales un número considerable de los pacientes del registro cumplirían esta clasificación, reforzando la necesidad 
de un enfoque multidisciplinario en el estudio de esta patología que debiese incluir la evaluación conjunta por neumólogos y reumatólogos.

Las principales limitaciones de este estudio corresponden al pequeño tamaño de la muestra $(n=30)$ junto al escaso número de pacientes con estudio histológico, el cual debiese haber sido considerado en el enfrentamiento diagnóstico en gran parte de los casos. A pesar de esto, se debe tener en cuenta que estas limitaciones son una realidad en la mayoría de los centros a nivel nacional; siendo fundamental exponer nuestra experiencia para continuar la investigación de la EPD en nuestro país.

\section{Bibliografía}

1.- MAHER T M. Diffuse parenchymal lung disease. Me-

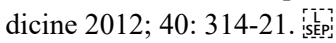

2.- KAUNISTO J, SALOMAA E R, HODGSON U, KAARTEENAHO R, MYLLÄRNIEMI M. Idiopathic pulmonary fibrosis -a systematic review on methodology for the collection of epidemiological data. BMC Pulm Med 2013; 13: 53.

3.- COULTAS D B, ZUMWALT R E, BLACK W C,
SOBONYA R E. The epidemiology of interstitial lung diseases. Am J Respir Crit Care Med 1994; 150: 967-72.

4.- AMERICAN THORACIC SOCIETY, EUROPEAN RESPIRATORY SOCIETY. Idiopathic pulmonary fibrosis: diagnosis and treatment: international consensus statement. Am J Respir Crit Care Med 2000; 161 (2 pt 1): 646-64.

5.- BAUMGARTNER K B, SAMET J M, STIDLEY C A, COLBY T V, WALDRON J A. Cigarette smoking: a risk factor for idiopathic pulmonary fibrosis. Am J Respir Care Med 1997; 155: 242-8.

6.- BEHR J, RYU J H. Pulmonary hypertension in interstitial lung disease. Eur Respir J 2008; 31: 1357-67.

7.- YUNT Z X, SOLOMON J J. Lung disease in rheumatoid Arthritis. Rheum Dis Clin N Am 2015,42: 225-36.

8.- $\quad$ ANDO M, MIYAZAKI E, ISHII T, MUKAI Y, YAMASUE M, FUJISAKI H, et al. Incidence of myeloperoxidase anti- neutrophil cytoplasmic antibody positivity and microscopic polyangitis in the course of idiopathic pulmonary fibrosis. Respir Med 2013; 107: 608-15.

9.- FISCHER A, ANTONIOU K M, BROWN K K, CADRANEL J, CORTE T J, DU BOIS R M, et al. An official European Respiratory Society/ American Thoracic Society research statement: interstitial pneumonia with autoimmune features. Eur Respir J 2015; 46: 976-87.
Correspondencia a:

Dr. José Miguel Chahuán S.

Hospital Dipreca

Vital Apoquindo 1200. Las Condes.

Región Metropolitana. Chile.

Email:josemiguel.chahuan@gmail.com 